\title{
Multiple effects of curcumin on promoting expression of the exon 7-containing SMN2 transcript
}

\author{
Dairong Feng ${ }^{1,2,3} \cdot$ Yi Cheng ${ }^{1,5} \cdot$ Yan Meng ${ }^{1,4} \cdot$ Liping Zou $^{4} \cdot$ Shangzhi Huang ${ }^{1} \cdot$ \\ Jiuyong $\mathrm{Xie}^{2,3}$
}

Received: 4 May 2015/Accepted: 14 August 2015/Published online: 19 September 2015

(C) Springer-Verlag Berlin Heidelberg 2015

\begin{abstract}
Survival of motor neuron 2 (SMN2) is a modifier gene for spinal muscular atrophy (SMA), a neurodegenerative disease caused by insufficient SMN protein mostly due to $S M N 1$ defect. $S M N 2$ is nearly identical to $S M N 1$ but unfortunately only able to produce a small amount of SMN protein due to exon 7 skipping. The exon 7-containing SMN2 transcript (SMN2_E7+) can be increased by a dietary compound, curcumin, but the involved molecular changes are not clear. Here we have found that in fibroblast cells of a SMA type II patient, curcumin enhanced the inclusion of $S M N 2$ exon 7. Examination of the potential splicing factors showed that curcumin specifically increased the protein and transcript levels of SRSF1. The increased SRSF1 protein
\end{abstract}

Electronic supplementary material The online version of this article (doi:10.1007/s12263-015-0486-y) contains supplementary material, which is available to authorized users.

\section{Shangzhi Huang}

hsz_pumc@ibms.pumc.edu.cn

$\triangle$ Jiuyong Xie

xiej@umanitoba.ca

1 Department of Medical Genetics, Institute of Basic Medical Sciences, Chinese Academy of Medical Sciences and Peking Union Medical College, Beijing 100005, China

2 Department of Physiology and Pathophysiology, College of Medicine, Faculty of Health Sciences, University of Manitoba, Winnipeg, MB R3E 0J9, Canada

3 Department of Biochemistry and Medical Genetics, College of Medicine, Faculty of Health Sciences, University of Manitoba, Winnipeg, MB R3E 0J9, Canada

4 Department of Pediatrics, Chinese PLA General Hospital, Beijing 100853, China

5 Present Address: Department of Diagnostic Ultrasound, Beijing Anzhen Hospital, Capital Medical University, Beijing 100029, China was mainly nuclear and hyperphosphorylated. Interestingly, the curcumin effects on the SMN2 and SRSF1 transcripts were inhibited by a protein deacetylase inhibitor, trichostatin A. Moreover, in support of its role in the SMN2 splicing, knocking down SRSF1 reduced the inclusion of $S M N 2$ exon 7. Thus, curcumin appears to have multiple effects on the $S M N 2$ transcript and its splicing regulators, including the change of alternative splicing and transcript/ protein level as well as phosphorylation. Protein deacetylases and phosphatases are likely involved in these effects. Interestingly, the effects all seem to favor production of the SMN2_E7+ transcript in SMA patient cells.

Keywords Curcumin - SMN2 - Splicing - SRSF1 · Phosphorylation

\section{Introduction}

Spinal muscular atrophy (SMA) is an autosomal recessive neuromuscular disorder with an estimated incidence of 1 in 6000 live births, representing the primary genetic cause of infant mortality (Monani 2005; Burghes and Beattie 2009). The disease is characterized by degeneration of $\alpha$-motor neurons in the anterior horn of the spinal cord and by consequent skeletal muscle atrophy (Monani 2005; Burghes and Beattie 2009). More than $96 \%$ of SMA patients have insufficient amount of the survival of motor neuron (SMN) protein due to the homozygous deletion of the SMN1 gene (Coovert et al. 1997; Lefebvre et al. 1995). Interestingly, a paralogous human gene SMN2 encodes the same but only a small amount of the SMN protein (Lefebvre et al. 1995; Kashima et al. 2007), due to its various extents of exon 7 skipping in different cells/tissues (Burnett et al. 2009; Lorson et al. 1998). Of the many SMN- 
deficient tissues (Zhang et al. 2008), spinal cord is the most affected in terms of function and survival (Burghes and Beattie 2009; Chen et al. 2008).

A C6-to-T transition in exon 7 of the SMN2 gene is shown to contribute to the skipping, by disrupting the binding of a splicing activator SRSF1 (serine-/arginine-rich splicing factor 1) (Cartegni and Krainer 2002; Cartegni et al. 2006) and (or) promoting the binding of a splicing repressor hnRNP A1 (heterogeneous nuclear ribonucleoprotein A1) (Kashima et al. 2007; Kashima and Manley 2003), in splicing reporter assays. Another splicing repressor of the SMN2 exon 7 is SAM68 (Src-associated substrate in Mitosis of $68 \mathrm{kDa}$ ) (Pedrotti et al. 2010). There are likely more factors involved in the regulation, as suggested by the effects of anti-sense oligonucleotides targeting other regions of the SMN2 pre-mRNA (Singh et al. 2006; Hua et al. 2010).

Though the expression of SMN2 is not sufficient to compensate for the homozygous loss of SMN1 (Lefebvre et al. 1995), multiple copies of SMN2 increase SMN protein level and inversely correlate with disease severity in SMA patients and transgenic mice (Hsieh-Li et al. 2000; Monani et al. 2000; Wirth et al. 2006; Swoboda et al. 2005). Thus, as a modifier gene for SMA, the alternative splicing of SMN2 exon 7 provides a promising target for SMA therapy (Hua et al. 2010).

Curcumin is a dietary polyphenol compound enriched in the turmeric root. It has been used in clinical trials of numerous human diseases (Gupta et al. 2013; Darvesh et al. 2012), likely involving its regulation of multiple targets including histone acetyl-transferase (Shishodia 2013; Shishodia et al. 2007). It has also been reported to increase the SMN2_E7+ transcript and SMN protein in fibroblast cells from a patient with SMA type I (Sakla and Lorson 2008); however, the underlying molecular changes have been unclear. For the treatment of human diseases (Gupta et al. 2012), it is necessary to identify its potential targets and effects.

In this study, we report that curcumin increases expression of the SMN2_E7+ transcript and SMN protein in fibroblast cells from a SMA patient with multiple effects: enhancing SMN2 exon 7 inclusion, increasing transcript/protein level, and phosphorylation of the splicing activator SRSF1. These effects likely involve deacetylases and phosphatases.

\section{Results}

\section{Curcumin increases the $\mathrm{SMN}$ protein and the proportion of $S M N 2 \_E 7+$ transcript in fibroblast cells form a patient with SMA type II}

To investigate potential curcumin effect on the expression of SMN protein and the usage of SMN2 exon 7, a SMA dermal fibroblast cell line (BJ301J) was established by using the skin biopsy of a patient (SMA type II, 6-month, male), who is deficient in both copies of the SMN1 gene but contains three copies of SMN2 (Figs. S1 and S2). In our initial tests, $15-25 \mu \mathrm{M}$ of curcumin increased the SMN2_E7+ transcript, but only $25 \mu \mathrm{M}$ was sufficient to upregulate the SMN protein level. We thus used $25 \mu \mathrm{M}$ of curcumin on these cells in the following experiments.

At $25 \mu \mathrm{M}$, curcumin efficiently increased the SMN protein level by 1.8 -folds after $48 \mathrm{~h}$ of treatment in the BJ301J cells (Fig. 1a, b, $p<0.001$ ). Consistent with the protein upregulation, the percentage of the SMN2_E7+ transcript was significantly increased from $45.0 \%( \pm 2.2 \%, n=3$, calculated at molar level, same as in the following) at $0 \mathrm{~h}$ to $59.1 \%( \pm 2.3 \%, n=3, p<0.01)$ at $24 \mathrm{~h}$, and further to $65.1 \%( \pm 0.9 \%, n=3, p<0.001)$ at $48 \mathrm{~h}$ (Fig. 1c, d) upon curcumin treatment.

\section{Curcumin-induced increase in the SMN2_E7+ variant is inhibited by trichostatin $A$, a deacetylase inhibitor}

Curcumin inhibits the histone acetyl-transferase (HAT) and phosphatases 2A and 5 (Balasubramanyam et al. 2004; Han et al. 2012). To examine whether protein deacetylation or phosphorylation is involved in the increase in SMN2_E7+ by curcumin, the BJ301J cells were pretreated for $2 \mathrm{~h}$ with trichostatin A (TSA, $1 \mu \mathrm{M}$ ), an inhibitor of deacetylase of histone and non-histone proteins (Dokmanovic et al. 2007; Glozak and Seto 2007), or okadaic acid (OA, $10 \mathrm{nM}$ ), an inhibitor of the protein Ser/Thr phosphatases PP1 and PP2A (Bialojan and Takai 1988), respectively, followed by curcumin treatment for $24 \mathrm{~h}$ (Fig. 2a). TSA but not OA pretreatment prevented the increase in the percentage of the SMN2_E7+ transcripts by curcumin in RT-PCR analysis (lane 3 vs. lane 2). Thus, curcumin-induced increase in the SMN2_E7+ variant is specifically inhibited by the protein deacetylase inhibitor TSA, suggesting that a deacetylation step is required for the regulation.

\section{SRSF1 is specifically increased at both protein and transcript levels by curcumin}

To identify the curcumin-regulated trans-acting factors, we examined several of them in the control of exon 7 usage, including SRSF1, hnRNP A1, and SAM68. The total protein level of SRSF1 was increased after 24-h treatment with curcumin in western blot analysis (Fig. 3a). The increase can also be clearly seen in the nucleus by immunostaining (Fig. 3b). In contrast, the SAM68 protein was reduced (Fig. 3a, b). Consistent with their protein changes, the level of SRSF1 and SAM68 mRNA transcripts was significantly increased by $144.6 \%( \pm 21.8 \%, n=3, p<0.01)$ or 
Fig. 1 Curcumin increases the SMN protein and the proportion of SMN2_E7+ transcript in fibroblast cells form a patient with SMA type II. a A Western blot of SMN protein levels in the cells treated with DMSO or curcumin for $48 \mathrm{~h}$, and $\mathbf{b}$ time course of the changes (mean $\pm \mathrm{SD}, n=3$, relative to $\beta$-ACTIN) as in a. c An agarose gel of RT-PCR products of the SMN2 variants (E7+ and E7-) in the cytoplasm, from the cells treated with curcumin in a time course, and $\mathbf{d}$ time course of the exon 7-containing SMN2 transcript changes (SMN2_E7+ $\mathrm{mol} \%$ ) calculated as the molar intensity of the 364-bp product $\left(S M N 2 \_\right.$E7+) relative to the total of the 364- and 310-bp (SMN2_E7-) products (mean $\pm \mathrm{SD}, n=3$ ) in each lane of the gel as in c. ${ }^{* *} p<0.01, * * * p<0.001$, compared to time 0
A

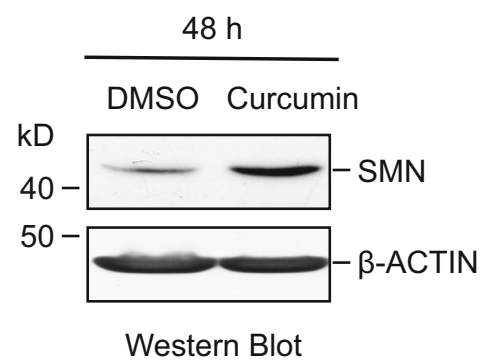

C

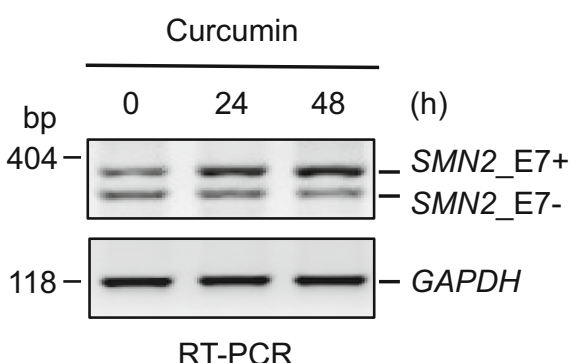

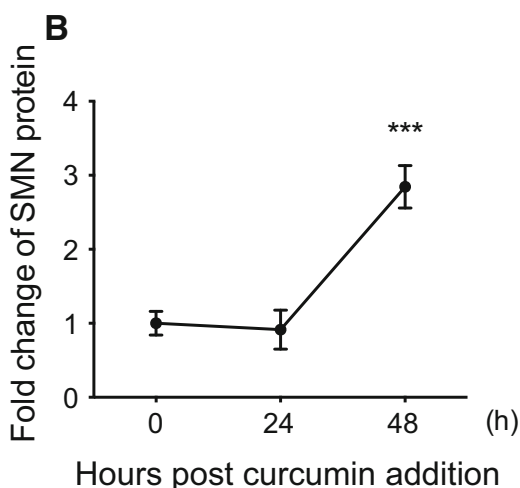

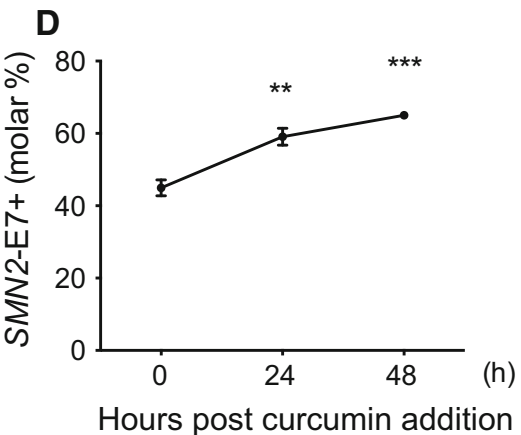

reduced by $19.8 \%( \pm 1.2 \%, n=3, p<0.001)$, respectively (Fig. 3c). HnRNP A1 did not change significantly in either protein level or transcript level (Fig. 3). Thus, SRSF1 and SAM68 are specifically regulated by curcumin at both protein and transcript levels.

\section{Curcumin increases the hyperphosphorylated isoform of SRSF1 in the nucleus}

To further characterize the changes of the splicing factors regulated by curcumin, we examined the phosphorylation status of nuclear SRSF1, which is required for SRSF1 nuclear/speckle localization and recruitment to nascent transcripts (Ngo et al. 2005). For this purpose, we used cleanly separated cytoplasmic and nuclear proteins, as indicated by the exclusive presence of GAPDH and hnRNP $\mathrm{F} / \mathrm{H}$ in the respective fractions (Fig. 4a, left panel). Analysis of these fractions indicated that the increase in total SRSF1 protein was mainly in the nucleus (right panel). Moreover, of the two bands below $35 \mathrm{kD}$ recognized by the SRSF1 antibody (clone 96), it was the upper band at about $34 \mathrm{kD}$ that became much stronger in the nucleus upon curcumin treatment. Thus, curcumin increases the level of a specific nuclear SRSF1 protein isoform.

The lower band of SRSF1 at about $32 \mathrm{kD}$ is clearly visible in the presence of $4 \mathrm{mM}$ of $\mathrm{NaF}$ in the protein lysates (Fig. 4a); however, it was not detectable when a higher concentration of $\mathrm{NaF}(10 \mathrm{mM})$ was used (Fig. 4b), suggesting that it is a less phosphorylated isoform. To explore the phosphorylation status of the two SRSF1 isoforms upon curcumin treatment, we immunoprecipitated (IP) the SRSF1 protein from the cytoplasmic fraction, followed by treatment with calf intestinal alkaline phosphatase (CIAP) (Fig. 4c). Upon CIAP treatment, the 34$\mathrm{kD}$ SRSF1 disappeared, compared to the untreated cell lysate or SRSF1 precipitate in western blot of the IP samples (lane 2 vs. lanes 1 and 3). Simultaneously, an extra much lower band appeared at about $30 \mathrm{kD}$ in the same CIAP-treated sample (lane 2). Thus, it is likely that the $\sim 34-\mathrm{kD}$ SRSF1 increased by curcumin is a hyperphosphorylated isoform.

\section{Curcumin-induced increase in SRSF1 is inhibited by trichostatin $\mathrm{A}$}

To see whether TSA also has effect on the curcumin regulation of SRSF1, we examined its protein and transcript levels in the BJ301J cells pretreated with deacetylase inhibitor TSA $(2 \mathrm{~h})$ followed by treatment with curcumin $(24 \mathrm{~h})$. SRSF1 protein was again increased in the nuclear fraction by curcumin addition (Fig. 5a). By contrast, curcumin-induced increase in the nuclear SRSF1 protein was reduced by TSA pretreatment (Fig. 5a). Accompanying the protein change, upregulation of the SRSF1 transcript 

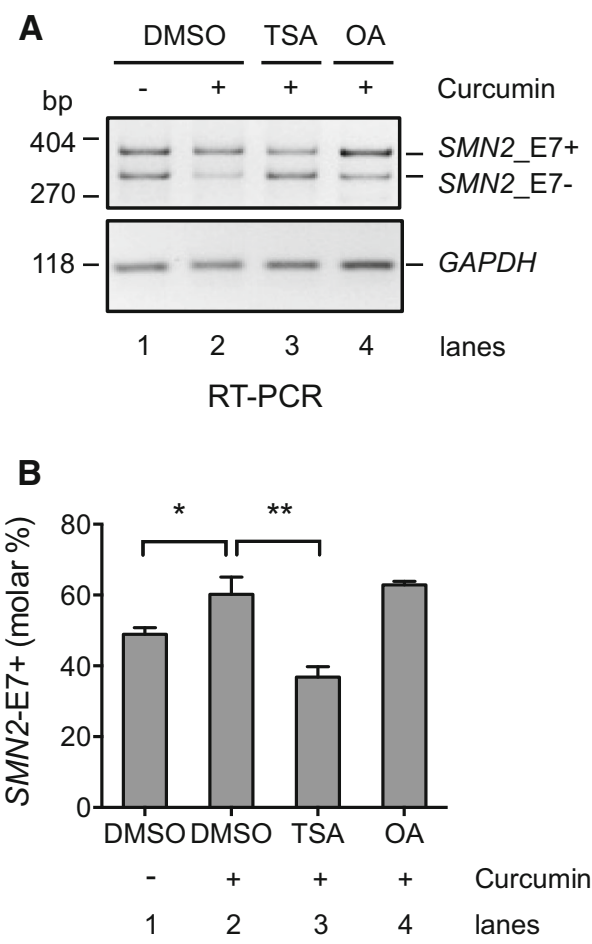

Fig. 2 Curcumin-induced increase in the SMN2_E7+ variant is inhibited by trichostatin A. a RT-PCR analysis of the $S M N 2$ variants (E7+ and E7-) in the cytoplasm of the BJ301J fibroblast cells upon $2 \mathrm{~h}$ of pretreatment with TSA or OA followed by 24-h addition of curcumin. GAPDH RNA loading control. b A bar graph represents the molar percentages of exon 7-containing SMN2 transcript $(S M N 2$ E7 $+\mathrm{mol} \%)$ are calculated as the molar intensity of the 364-bp product (SMN2_E7+) relative to the total of the 364- and 310-bp (SMN2_E7-) products (mean $\pm \mathrm{SD}, n=3$ ) in each lane of the gel in a. $* p<0.05, * * p<0.01$, comparison between the indicated samples

$(244.6 \pm 21.9 \%, n=3, p<0.01$, Fig. $5 b)$ by curcumin in comparison with DMSO was abolished upon pretreatment by TSA $(117.0 \pm 11.4 \%, n=3, p<0.001$, Fig. 5b). Therefore, a protein deacetylation step is likely also required for the curcumin-induced increase in SRSF1 transcript/protein as well.

\section{SRSF1 knockdown reduces the inclusion of SMN2 exon 7}

To determine the role of SRSF1 in SMN2 exon 7 usage and in the curcumin effect, we carried out lentiviral vectormediated shRNA knockdown of SRSF1 in the BJ301J cells (Fig. 6a). In mock-treated or scrambled shRNA-expressing cells, the molar percentage of the SMN2_E7+ variant was increased from about $43.7-56.1 \%$ by curcumin (Fig. 6 b, upper panel, lanes 1-4). Upon SRSF1 knockdown, the SMN2_E7+ transcript level was reduced significantly to $32.5 \%( \pm 1.4 \%, n=3, p<0.01$, lane 5 vs. lanes 1 and 3 ). Treatment with curcumin was still able to increase the level but merely to $44.0 \%( \pm 1.9 \%, n=3$, lane 6$)$, significantly lower than that in control samples $(p<0.001$, lanes 2 and 4). In contrast, hnRNP A1 knockdown did not change the basal level of the SMN2 splice variants (lanes 7-8). Thus, SRSF1 knockdown reduces the percentage of exon 7 inclusion in the SMA patient cells, supporting SRSF1 as a weak splicing activator of SMN2 exon 7. However, it is not essential for the curcumin enhancement of exon 7 inclusion, suggesting the existence of more factors involved in the curcumin effect.

Taken together, these data indicate that the dietary compound curcumin has multiple effects on SMN2 and its splicing regulators to promote the expression of the SMN2_E7+ transcripts in SMA fibroblast cells. These effects likely involve protein deacetylation and/or phosphorylation.

\section{Discussion}

SMA patient fibroblast cells from a type I patient have been used to investigate the regulation of SMN2 pre-mRNA splicing by small compounds (Sakla and Lorson 2008; Dayangac-Erden et al. 2011). In this study, the BJ301J cells were derived from a patient (male, 9 months old) with SMA type II. They are homozygously deleted of the SMNI but have three copies of the SMN2 gene (Fig. S1), with one more copy of SMN2 than the previously reported GM3813 cells (from a SMA type I, 3-year old male) (Sakla and Lorson 2008; Dayangac-Erden et al. 2011). In both types of cells, the fold increases in the percentages of SMN2_E7+ transcripts are similar (about 1.5-fold) upon 24-h treatment with $25 \mu \mathrm{M}$ of curcumin. This similar change implies that the curcumin targets in promoting the production of fulllength SMN2 transcript might be the same in different sources and types of SMA cells.

Curcumin has pleiotropic effects on a number of targets in cells (Shishodia 2013; Shishodia et al. 2007), particularly the histone acetyltransferase p300-/CREB-binding protein (p300/CBP) (Balasubramanyam et al. 2004). Of the different effects by curcumin, changes in splicing and transcript levels (Figs. 1, 2, 3) could be contributed by its inhibition of $\mathrm{p} 300 / \mathrm{CBP}$ and protein/histone acetylation (Balasubramanyam et al. 2004). This is supported by the inhibition of these curcumin effects by the histone deacetylase (HDAC) inhibitor TSA (Figs. 2, 5). Particularly for SRSF1, we have shown that curcumin inhibits the histone acetylation/transcription of factors involved in the nonsense-mediated decay (NMD) pathway, consequently increasing the level of NMD-targeted SRSF1 variant transcripts in cells (Feng et al. 2015). The HDAC inhibitor may also increase the processivity of RNA Polymerase II and reduce co-transcriptional association of splicing 
A
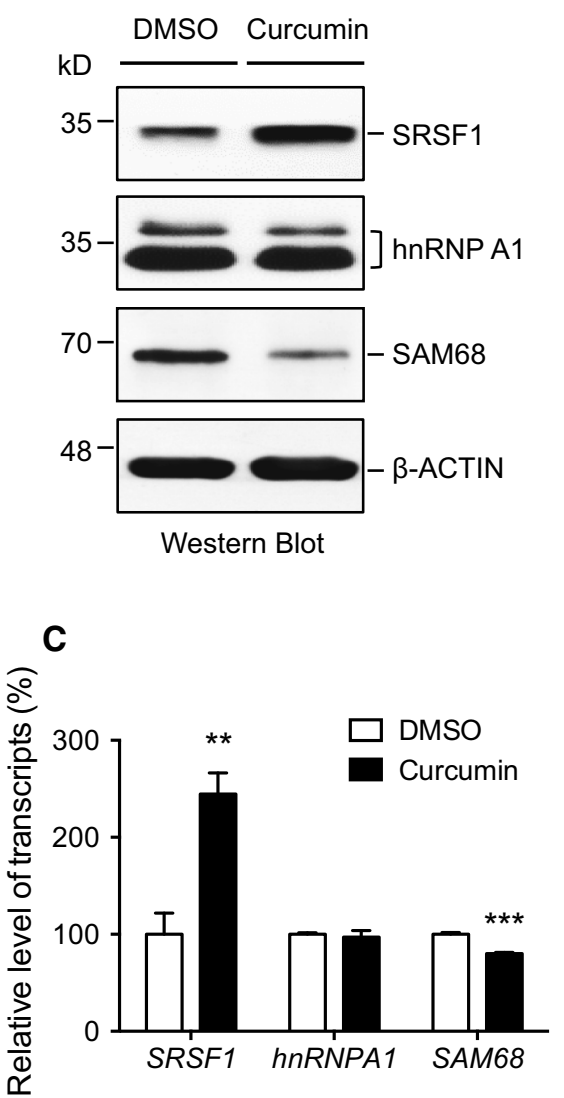

B

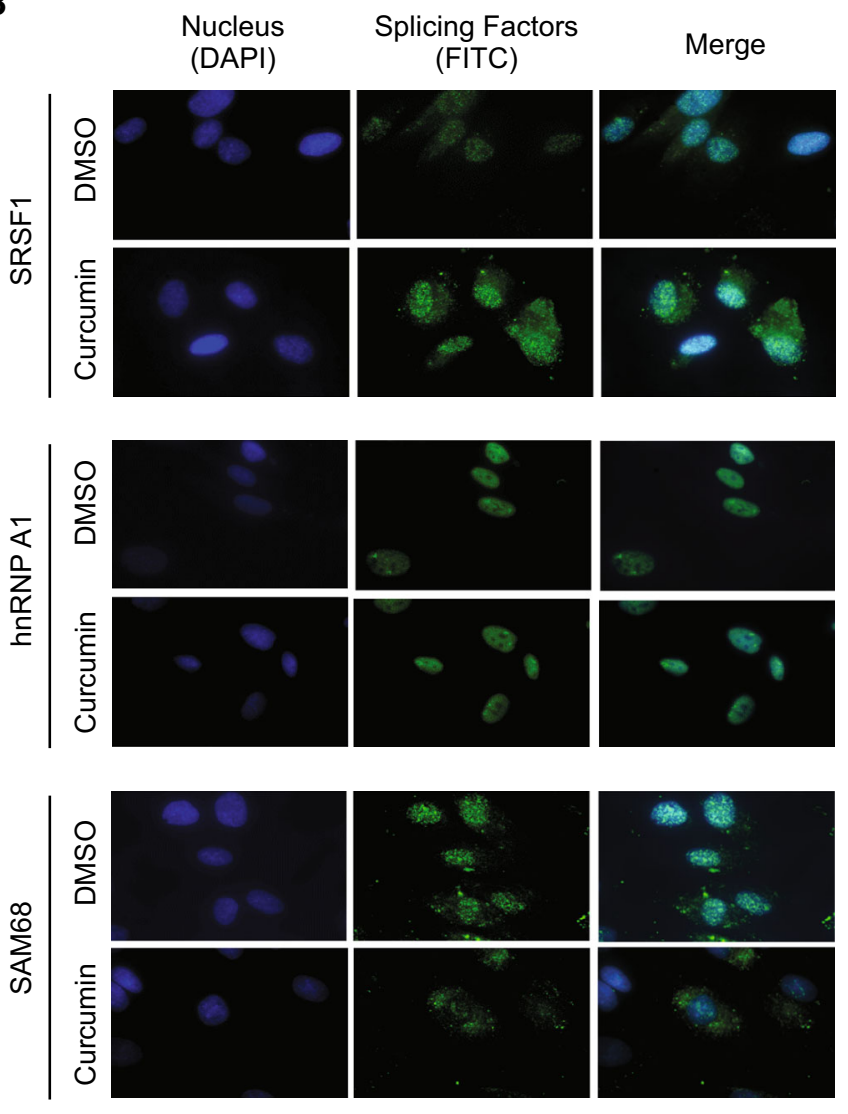

Fig. 3 SRSF1 is specifically increased by curcumin. a Representative western blots of total proteins of splicing factors involved in the regulation of the SMN2 exon 7 alternative splicing upon 24-h treatment of BJ301J cells with DMSO or curcumin. $\beta$-ACTIN proteinloading control. b Immunostaining of splicing factors (green) in the BJ301J cells upon 24-h treatment with DMSO or curcumin. Nuclei

regulators with certain alternative exons (Hnilicova et al. 2011), which may counteract the curcumin-enhanced exon 7 usage.

The curcumin effect on SMN2 splicing and SRSF1 phosphorylation (Figs. 1, 2, 4) may also involve the inhibition of phosphatases such as PP1 and PP2A. PP1 binds to the beta- 4 sheet of the SRSF1 RNA recognition motif (RRM1) domain, thus dephosphorylating the splicing factor (Novoyatleva et al. 2008). PP1 inhibition promotes SMN2 exon 7 inclusion in fibroblast cells of SMA patients and spinal cord of SMA mice (Novoyatleva et al. 2008). Both PP1 and PP2A can be inhibited by okadaic acid (OA). PP2A activity can also be inhibited by curcumin (Han et al. 2012). However, in our experiments, OA pretreatment did not change the curcumin effect on exon 7 inclusion in cells (Fig. 2). A reasonable explanation is that curcumin and OA have overlapping effects on the phosphatases and thus the are stained with DAPI (blue). c A bar graph of the transcript levels by RT-PCR analysis of the splicing factors (mean $\pm \mathrm{SD}, n=3$ ) upon $24 \mathrm{~h}$ of treatment with DMSO or curcumin. Each transcript was normalized to that of $G A P D H$ and then to that of the DMSO-treated sample, which is set as $100 \%$. **p $<0.01, * * * p<0.001$, compared to DMSO-treated samples

phosphorylation of the splicing factors for SMN2 exon 7 usage.

In the immunoprecipitation experiment (Fig. 4c), we were not able to efficiently immunoprecipitate the nuclear SRSF1 protein, likely due to occupation of its RRM1 domain by phosphatases (Novoyatleva et al. 2008), which is part of the N-terminal antigen targeted by the SRSF1 antibody.

In summary, we have observed multiple effects of curcumin on the expression of genes involved in the production of the full-length SMN2 protein. These effects on gene transcription/splicing and protein phosphorylation could be contributed by the inhibitory effect of curcumin on the histone acetyl-transferase $\mathrm{p} 300 / \mathrm{CBP}$ and protein phosphatases. These two targets are likely important for the overall beneficial effects for producing the full-length SMN2 protein. 

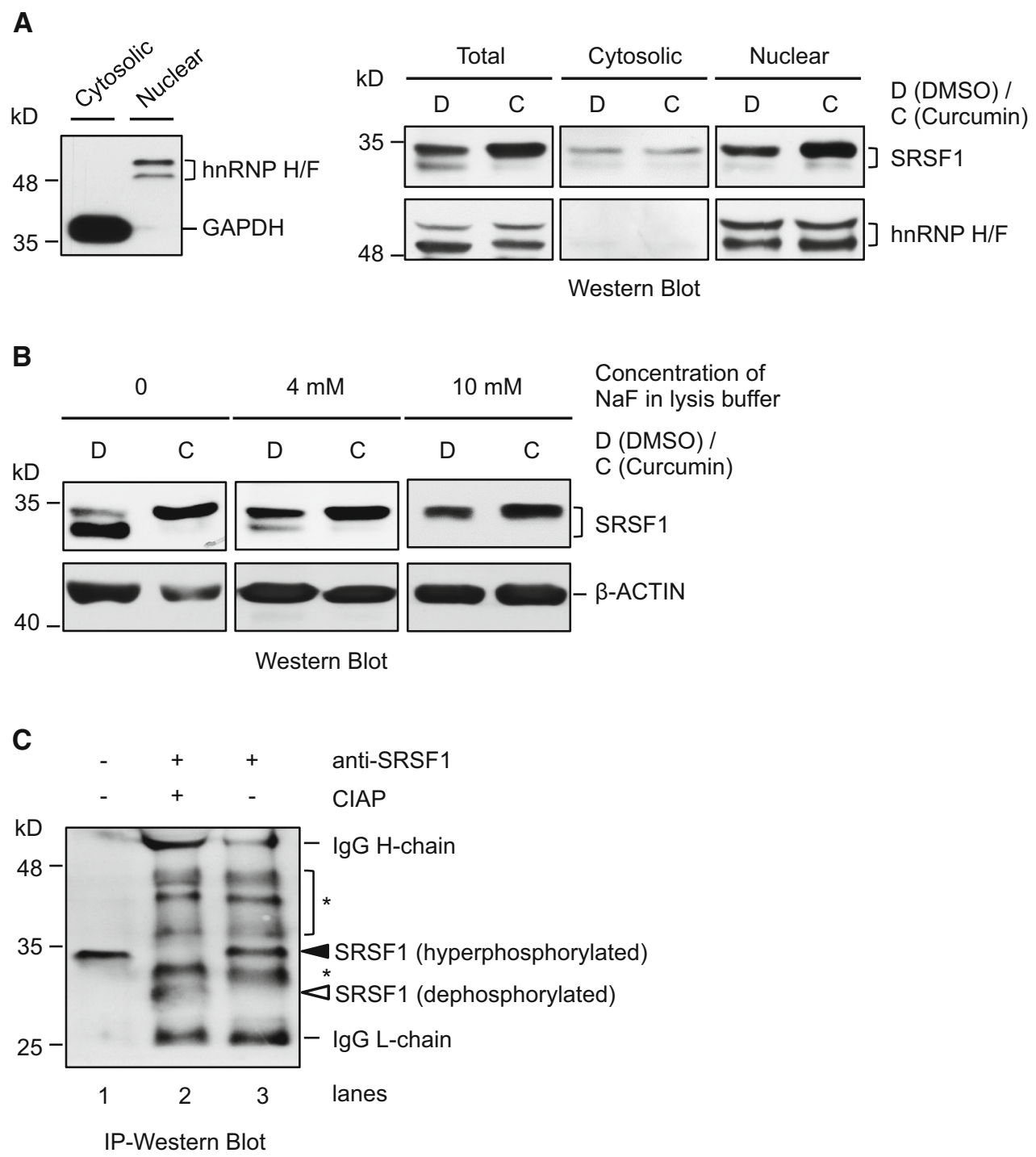

Fig. 4 Curcumin increases the hyperphosphorylated isoform of SRSF1 in the nucleus. a Left panel Western blotting analysis of the fractionation of cytosol and nuclei of the BJ301J cells, GAPDH and hnRNP H/F are as the specific markers for cytosolic and nuclear fractions, respectively. Right panel Western blotting analysis of the expression of SRSF1 in total lysates (T), cytosolic (C) and nuclear (N) fractions of the BJ301J cells upon 24-h treatment with DMSO or $25 \mu \mathrm{M}$ of curcumin. hnRNP $H / F$ protein loading control. Two bands are shown in all of the lysate samples, both of them can be recognized by SRSF1 antibody (clone 96). b Western blotting analysis of SRSF1

\section{Materials and methods}

\section{Cell culture and treatment}

Human fibroblast cells (BJ301J) were derived from a type II SMA patient (male, 9 months old) with three copies of the SMN2 gene and maintained in Dulbecco's modified Eagle's medium supplemented with $10 \%$ fetal protein isoforms in total lysates supplemented with different concentrations of NaF. The lower band of SRSF1 upon curcumin treatment is relatively much weaker than that in DMSO-treated cell lysate and not detected when a higher concentration of $\mathrm{NaF}(10 \mathrm{mM})$ was used. $\beta$-ACTIN protein loading control. c Western blot analysis of IP cytoplasmic SRSF1 protein in curcumin-treated BJ301J fibroblast cells with or without CIAP treatment. Black-filled and unfilled triangles indicate the hyperphosphorylated and the dephosphorylated isoforms of SRSF1, respectively. *Non-specific bands

bovine serum, $2 \mathrm{mM}$ glutamine, and $1 \%$ penicillinstreptomycin solution (Invitrogen) at $37{ }^{\circ} \mathrm{C}$ in a humidified atmosphere containing $5 \% \mathrm{CO}_{2}$. Cells were treated with DMSO or $25 \mu \mathrm{M}$ of curcumin (Sigma-Aldrich) for 0,24 , or $48 \mathrm{~h}$. In the assay of inhibitors on transcript of $S M N 2$ or SRSF1, cells were pretreated with each inhibitor for $2 \mathrm{~h}$ followed by 24-h addition of DMSO or $25 \mu \mathrm{M}$ of curcumin. 
A

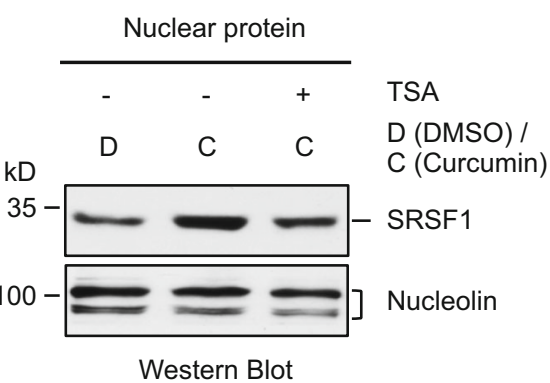

B
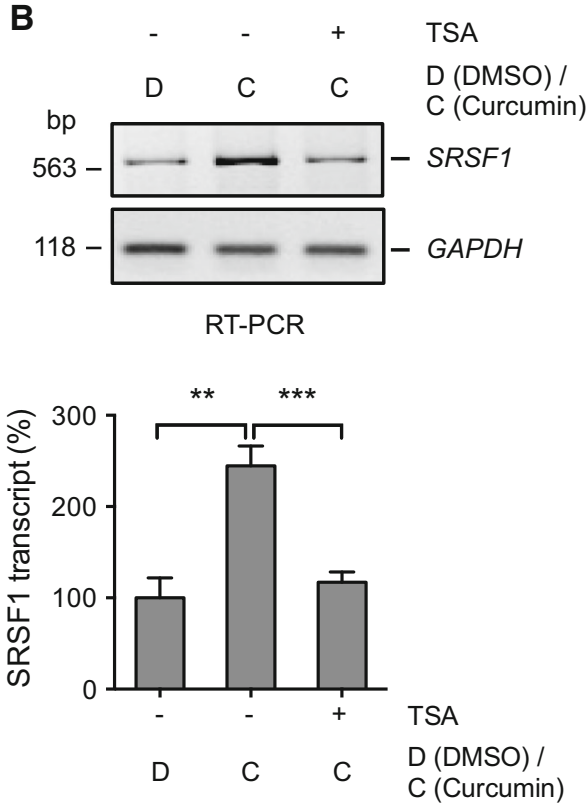

Fig. 5 Curcumin-induced increase in SRSF1 is inhibited by trichostatin A (TSA). a Western blots of SRSF1 protein in the nuclei of the BJ301J cells upon $2 \mathrm{~h}$ of pretreatment with or without TSA, followed by 24-h addition of DMSO or curcumin. Nucleolin nuclear protein loading control. b Upper panel RT-PCR analysis of the SRSF1 transcripts in the cytoplasm of the BJ301J fibroblast cells upon $2 \mathrm{~h}$ of pretreatment with or without TSA, followed by 24-h addition of DMSO or curcumin. GAPDH RNA loading control. Lower panel A bar graph of the $S R S F 1$ transcript changes (mean $\pm \mathrm{SD}, n=3$ ) as in the upper panel. SRSF1 transcript was normalized to that of GAPDH and then to that of the sample treated with DMSO but not TSA (-), which is set as $100 \%$. **p $<0.01$, ***p $<0.001$, comparison between the indicated samples

\section{Knockdown of splicing factors using lentiviral vector-mediated transduction}

Lentiviral particles were prepared using the shRNA plasmids pGIPZ-shSRSF1 (Open Biosystems, RMM443199938975, 5'-TCG AGA TCG AGA TCT TCC A-3'), pGIPZ-shhnRNPA1 (5'-GTG TAA AGC ATT CCA ACG A-3'), and pGIPZ-scrambled (5'-TAG TGA AGC CAC AGA ATA T- $3^{\prime}$ ) according to our previous procedures (Liu et al. 2012; Yu et al. 2009). Cells were treated with DMSO
A

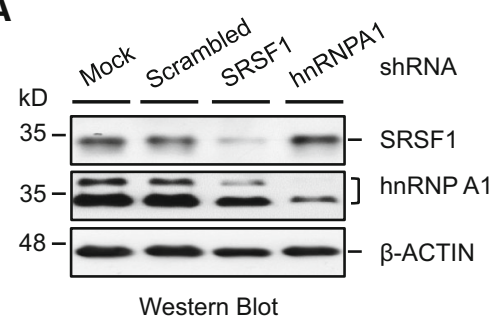

B
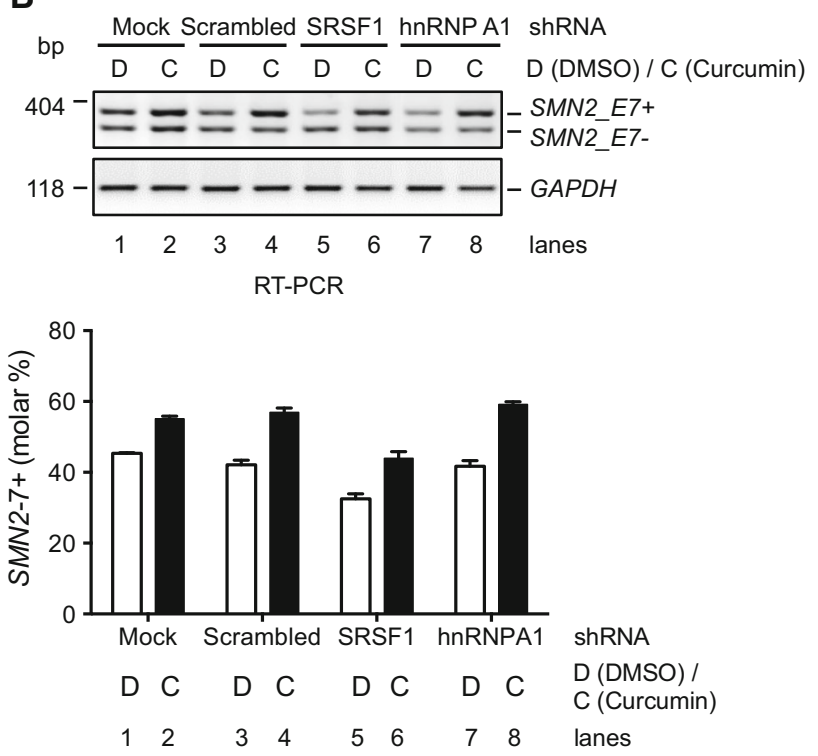

Fig. 6 SRSF1 knockdown reduces the inclusion of SMN2 exon 7. a Western blots of SRSF1 or hnRNP A1 from the BJ301J cells 7 days after transduction of lentiviral particles containing interfering shRNA. $\beta$-ACTIN protein loading control. b Upper panel Agarose gels of RTPCR products of the SMN2 variants in the cytoplasm of the BJ301 cells knocked down of indicated factors. Cells were treated for $24 \mathrm{~h}$ with DMSO or curcumin 7 days after transduction. GAPDH RNA loading control. Lower panel A bar graph of the molar percentages of exon 7-containing SMN2 transcript (SMN2_E7+), calculated as the molar intensity of the 364-bp product (SMN2_E7+) relative to the total of the 364- and 310-bp (SMN2_E7-) products (mean $\pm \mathrm{SD}, n=3$ ) in each lane of the gel shown in the upper panel

or curcumin 7 days after transduction. The silencing effects were confirmed by RT-PCR and immunoblotting.

\section{Reverse transcription polymerase chain reaction}

Cytoplasmic RNA was fractionated according to our previous procedure (Feng et al. 2015; Ma et al. 2007) and extracted using the GenElute Mammalian Total RNA Miniprep Kit (Sigma-Aldrich). One microgram of cytoplasmic RNA was included in a $10 \mu \mathrm{l}$ reverse transcription reaction. PCR reactions were carried out for 26-30 cycles. The sequences $\left(5^{\prime}-3^{\prime}\right)$ of the primer pairs were as follows, with the forward primer listed first followed by the reverse primer for each gene. SMN2: AAG ACT GGG ACC AGG 
AAA GC, TAT CTT CTA TAA CGC TTC ACA TTC CAG; SRSF1: CCT CCA GAC ATC CGA ACC AAG, TGC TAC GGC TTC TGC TAC GAC; hnRNP Al: GTC TAA GTC AGA GTC TCC TAA AGA GCC, TCT CAT TAC CAC ACA GTC CGT G; SAM68: GCT GAC GGC AGA AAT TGA GAA G, TTG ACA GGT ATC AGC ACT CGC TC; GAPDH: GTC AAC GGA TTT GGT CGT ATT G, AAC CAT GTA GTT GAG GTC AAT GAA G.

PCR products were resolved in 2-3\% agarose gels containing $0.5 \mu \mathrm{g} / \mathrm{ml}$ ethidium bromide. The gels presented in figures are inverted digital images. The abundance of the SMN2_E7+ splice variants is expressed as molar percentages relative to the total of the SMN2 variants $(\mathrm{E} 7+$ and E7-)

\section{Western blotting}

Cells were rinsed three times with ice-cold PBS, harvested using cell scrapers, pelleted by centrifugation at $14,000 \mathrm{rpm}$ for $30 \mathrm{~s}$ at $4{ }^{\circ} \mathrm{C}$, and lysed in RIPA buffer (containing $2 \mathrm{mM}$ PMSF, $2 \mathrm{mM} \mathrm{Na}_{3} \mathrm{VO}_{4}$ and $10 \mathrm{mM} \mathrm{NaF}$ ) (Feng et al. 2015). Protein was quantified using the Bradford method, and samples were run on 10 or $12 \%$ Tris-glycine acrylamide gels and then transferred to polyvinylidene fluoride membranes. The membranes were blocked in $5 \%$ dry milk and probed with the following mouse antibodies, which were all purchased from SantaCruz Biotechnology unless otherwise indicated: anti-SMN (H-7, 1:400), anti-SRSF1 (clone 96, 1:500), anti-hnRNP A1 (9H10, 1:1000), anti-SAM68 (7-1, 1:250), anti-nucleolin (H-6, 1:1000), anti- $\beta$-actin (C4, 1:1000), and antiGAPDH (Sigma, 1G5, 1:2000). After incubation with peroxidase-conjugated goat anti-mouse immunoglobulin $\mathrm{M}$ or G secondary antibodies (Sigma-Aldrich, 1:2000), proteins were visualized using enhanced chemiluminescence (GE Healthcare). Densitometry of the resulting bands was analyzed by Image $\mathbf{J}$ (developed by the U.S. National Institutes of Health and available at http://rsb.info.nih.gov/ $\mathrm{ij} /)$.

\section{Immunostaining}

BJ301J fibroblast cells were plated over the slides in sixwell plates, treated with DMSO or curcumin for $24 \mathrm{~h}$, rinsed twice in ice-cold phosphate-buffered saline (PBS) with $1 \%$ BSA, fixed with $4 \%$ paraformaldehyde (PFA) for $15 \mathrm{~min}$ and then permeabilized with $0.2 \%$ Triton $\mathrm{X}-100$ for $10 \mathrm{~min}$ at room temperature. The fixed cells were incubated overnight at $4{ }^{\circ} \mathrm{C}$ with mouse monoclonal antibodies anti-SRSF1, anti-hnRNP A1, and anti-Sam68, respectively. The primary antibodies were diluted at 1:100 in TBS $(20 \mathrm{mM}$ TrisCl, $500 \mathrm{mM} \mathrm{NaCl}$ ) containing $1 \%$ BSA. Cells were rinsed twice with TBS and incubated with goat anti-mouse fluorescent secondary antibody (conjugated with FITC, 1:1000) in the dark for $1 \mathrm{~h}$ at room temperature. Cell nuclei were counterstained with DAPI (1:6000). The stained cells were mounted with mounting media (Sigma-Aldrich). Images were taken at $100 \times$ magnification with an Olympus microscope.

\section{Fractionation of nuclear and cytoplasmic proteins}

BJ301J fibroblast cells were rinsed with ice-cold PBS three times in the dishes then harvested in $1 \mathrm{ml}$ of ice-cold PBS using rubber scrapers. Cell pellets were collected into 1.5$\mathrm{ml}$ tubes by centrifuging at $14,000 \mathrm{rpm}$ for $30 \mathrm{~s}$ and then resuspended in ice-cold NP-40 buffer supplemented with $2 \mathrm{mM}$ PMSF, $2 \mathrm{mM} \mathrm{Na} \mathrm{VO}_{4}$ and $10 \mathrm{mM} \mathrm{NaF}$. After centrifugation at $14,000 \mathrm{rpm}$ for $2 \mathrm{~min}$, the supernatant was used as cytoplasmic fraction by additional centrifugation at 14,000 rpm through $24 \%$ (w/v) sucrose cushion, the nuclear pellets were washed twice using $1 \mathrm{ml}$ of icecold NP-40 buffer followed by resuspension in RIPA buffer supplemented with $2 \mathrm{mM}$ PMSF, $2 \mathrm{mM} \mathrm{Na} \mathrm{VO}_{4}$ and $10 \mathrm{mM} \mathrm{NaF}$, then used as nuclear fraction after sonication.

\section{Immunoprecipitaion and phosphatase assay}

BJ301J fibroblast cells were rinsed with cold PBS for three times. The cytoplasmic and nuclear lysates were prepared according to the protocol described in the section of "Cytoplasm and nuclei fractionation." Protein G beads were washed with cold PBS for five times, then packed with $2 \mu \mathrm{g}$ of anti-SRSF1 antibody and incubated at $4{ }^{\circ} \mathrm{C}$ under rotary agitation for $4 \mathrm{~h}$. After washing with cold PBS, the packed beads were incubated overnight with lysates at $4{ }^{\circ} \mathrm{C}$. When the incubation time was over, the supernatant was removed by centrifugation, and the beads were washed in lysis buffer three times for further analysis.

The precipitations were suspended in $1 \times$ reaction buffer with 10 units of CIAP and incubated at $37{ }^{\circ} \mathrm{C}$ for $60 \mathrm{~min}$. After that, the suspensions were mixed with $6 \times$ SDS loading buffer, heated at $95{ }^{\circ} \mathrm{C}$ for $5 \mathrm{~min}$ to denature the proteins, and separated them from the protein $\mathrm{G}$ beads, and the supernatants were used for western blotting analysis.

\section{Statistical analysis}

Data were analyzed by two-tailed Student's $t$ test. A $p$ value $<0.05$ was considered significant.

Acknowledgments We sincerely thank the SMA patient and his family for donating the skin biopsy sample, Ms. Weimin Zhang for help establishing the primary culture of BJ301J, Dr. Guodong Liu for providing the shhnRNP A1 plasmid, Drs. Aleh Razanau and Wenguang Cao for helpful discussions, and Lei Lei and Nan Liang for 
proofreading the manuscript. This work was supported by a Manitoba Research Chair Fund and in part by a Canadian Institutes of Health Research (CIHR) Operating Grant FRN_106608 to JX, by FRN_2006BIA05A07 and 2006BIA05A08 from the National Key Technology R\&D Program of China to SH.

\section{Compliance with ethical standards}

Conflict of interest All of the authors declare no conflict of interests.

Ethical standards All procedures performed in this study involving the SMA patient were in accordance with the ethical standards of the institutional and/or national research committee and with the 1964 Declaration of Helsinki and its later amendments or comparable ethical standards. Informed consent was obtained from the SMA patient included in the study.

\section{References}

Balasubramanyam K et al (2004) Curcumin, a novel p300/CREBbinding protein-specific inhibitor of acetyltransferase, represses the acetylation of histone/nonhistone proteins and histone acetyltransferase-dependent chromatin transcription. J Biol Chem 279(49):51163-51171

Bialojan C, Takai A (1988) Inhibitory effect of a marine-sponge toxin, okadaic acid, on protein phosphatases. Specificity and kinetics. Biochem J 256(1):283-290

Burghes AH, Beattie CE (2009) Spinal muscular atrophy: why do low levels of survival motor neuron protein make motor neurons sick? Nat Rev Neurosci 10(8):597-609

Burnett BG et al (2009) Regulation of SMN protein stability. Mol Cell Biol 29(5):1107-1115

Cartegni L, Krainer AR (2002) Disruption of an SF2/ASF-dependent exonic splicing enhancer in SMN2 causes spinal muscular atrophy in the absence of SMN1. Nat Genet 30(4):377-384

Cartegni L et al (2006) Determinants of exon 7 splicing in the spinal muscular atrophy genes, SMN1 and SMN2. Am J Hum Genet 78(1):63-77

Chen HH et al (2008) The RNA binding protein hnRNP Q modulates the utilization of exon 7 in the survival motor neuron 2 (SMN2) gene. Mol Cell Biol 28(22):6929-6938

Coovert DD et al (1997) The survival motor neuron protein in spinal muscular atrophy. Hum Mol Genet 6(8):1205-1214

Darvesh AS et al (2012) Curcumin and neurodegenerative diseases: a perspective. Expert Opin Investig Drugs 21(8):1123-1140

Dayangac-Erden D et al (2011) Carboxylic acid derivatives of histone deacetylase inhibitors induce full length SMN2 transcripts: a promising target for spinal muscular atrophy therapeutics. Arch Med Sci 7(2):230-234

Dokmanovic M, Clarke C, Marks PA (2007) Histone deacetylase inhibitors: overview and perspectives. Mol Cancer Res 5(10): 981-989

Feng D et al (2015) Increase of a group of PTC $(+)$ transcripts by curcumin through inhibition of the NMD pathway. Biochim Biophys Acta 1849(8):1104-1115

Glozak MA, Seto E (2007) Histone deacetylases and cancer. Oncogene 26(37):5420-5432

Gupta SC et al (2012) Discovery of curcumin, a component of golden spice, and its miraculous biological activities. Clin Exp Pharmacol Physiol 39(3):283-299

Gupta SC, Patchva S, Aggarwal BB (2013) Therapeutic roles of curcumin: lessons learned from clinical trials. AAPS J 15(1):195-218
Han X et al (2012) Curcumin inhibits protein phosphatases 2A and 5, leading to activation of mitogen-activated protein kinases and death in tumor cells. Carcinogenesis 33(4):868-875

Hnilicova $\mathbf{J}$ et al (2011) Histone deacetylase activity modulates alternative splicing. PLoS One 6(2):e16727

Hsieh-Li HM et al (2000) A mouse model for spinal muscular atrophy. Nat Genet 24(1):66-70

Hua Y et al (2010) Antisense correction of SMN2 splicing in the CNS rescues necrosis in a type III SMA mouse model. Genes Dev 24(15):1634-1644

Kashima T, Manley JL (2003) A negative element in SMN2 exon 7 inhibits splicing in spinal muscular atrophy. Nat Genet 34(4):460-463

Kashima $\mathrm{T}$ et al (2007) hnRNP A1 functions with specificity in repression of SMN2 exon 7 splicing. Hum Mol Genet 16(24):3149-3159

Lefebvre $S$ et al (1995) Identification and characterization of a spinal muscular atrophy-determining gene. Cell 80(1):155-165

Liu G et al (2012) A conserved serine of heterogeneous nuclear ribonucleoprotein L ( $\mathrm{hnRNP} \mathrm{L}$ ) mediates depolarization-regulated alternative splicing of potassium channels. J Biol Chem 287(27):22709-22716

Lorson CL et al (1998) SMN oligomerization defect correlates with spinal muscular atrophy severity. Nat Genet 19(1):63-66

Ma S et al (2007) Relocalization of the polypyrimidine tract-binding protein during PKA-induced neurite growth. Biochim Biophys Acta 1773(6):912-923

Monani UR (2005) Spinal muscular atrophy: a deficiency in a ubiquitous protein; a motor neuron-specific disease. Neuron 48(6):885-896

Monani UR et al (2000) The human centromeric survival motor neuron gene (SMN2) rescues embryonic lethality in $\mathrm{Smn}(-/-)$ mice and results in a mouse with spinal muscular atrophy. Hum Mol Genet 9(3):333-339

Ngo JC et al (2005) Interplay between SRPK and Clk/Sty kinases in phosphorylation of the splicing factor ASF/SF2 is regulated by a docking motif in ASF/SF2. Mol Cell 20(1):77-89

Novoyatleva $T$ et al (2008) Protein phosphatase 1 binds to the RNA recognition motif of several splicing factors and regulates alternative pre-mRNA processing. Hum Mol Genet 17(1):52-70

Pedrotti S et al (2010) The splicing regulator Sam68 binds to a novel exonic splicing silencer and functions in SMN2 alternative splicing in spinal muscular atrophy. EMBO J 29(7):1235-1247

Sakla MS, Lorson CL (2008) Induction of full-length survival motor neuron by polyphenol botanical compounds. Hum Genet 122(6):635-643

Shishodia S (2013) Molecular mechanisms of curcumin action: gene expression. BioFactors 39(1):37-55

Shishodia S, Singh T, Chaturvedi MM (2007) Modulation of transcription factors by curcumin. Adv Exp Med Biol 595:127-148

Singh NK et al (2006) Splicing of a critical exon of human Survival Motor Neuron is regulated by a unique silencer element located in the last intron. Mol Cell Biol 26(4):1333-1346

Swoboda KJ et al (2005) Natural history of denervation in SMA: relation to age, SMN2 copy number, and function. Ann Neurol 57(5):704-712

Wirth B et al (2006) Mildly affected patients with spinal muscular atrophy are partially protected by an increased SMN2 copy number. Hum Genet 119(4):422-428

$\mathrm{Yu} \mathrm{J}$ et al (2009) The heterogeneous nuclear ribonucleoprotein $\mathrm{L}$ is an essential component in the $\mathrm{Ca} 2+/$ calmodulin-dependent protein kinase IV-regulated alternative splicing through cytidineadenosine repeats. J Biol Chem 284(3):1505-1513

Zhang Z et al (2008) SMN deficiency causes tissue-specific perturbations in the repertoire of snRNAs and widespread defects in splicing. Cell 133(4):585-600 